\title{
TRIPLE COHOMOLOGY AND DIVIDED POWERS ALGEBRAS IN PRIME CHARACTERISTIC
}

\author{
IOANNIS DOKAS
}

(Received 6 November 2007; accepted 27 August 2008)

Communicated by J. Du

\begin{abstract}
In this paper using the Quillen-Barr-Beck (co-)homology theory of universal algebras we define (co-)homology groups for commutative algebras with divided powers in prime characteristic. In particular, we determine for $A$ a commutative $\mathbb{F}_{p}$-algebra with divided powers, the category of Beck $A$-modules and the group of Beck derivations. We construct the abelianization functor and we define (co-)homology. Moreover, we determine the cohomology in low dimensions and we interpret the first cohomology in terms of extensions.
\end{abstract}

2000 Mathematics subject classification: primary 18C15; secondary 13D03, 18 G60.

Keywords and phrases: commutative algebras with divided powers, Quillen-Barr-Beck cohomology, triples.

\section{Introduction}

Cartan in [2] defined the structure of divided powers algebras and proved that the homotopy of a simplicial commutative algebra is equipped with divided powers operations. In Section 2, we recall some preliminary results and we point out the fact that in prime characteristic, by the theorems of Cartan and Soublin [9], the system of divided powers operations is determined by the $p$ th operation.

In Section 3, we determine the category of Beck modules and derivations. In particular, if $\mathrm{p}$-Com denotes the category of commutative $\mathbb{F}_{p}$-algebras with divided powers, we determine for $A \in \mathrm{p}$-Com the category of abelian group objects $(\mathrm{p}-\mathrm{Com} / A)_{\mathrm{ab}}$ of the slice category $\mathrm{p}-\mathrm{Com} / A$. Then, for a divided powers algebra $B \in \mathrm{p}-\mathrm{Com} / A$ we determine the $\operatorname{group} \operatorname{Der}_{p}(B, M)$ of Beck derivations of $B$ to a Beck $A$-module $M$.

In Section 4, by analogy with the definition of the Kähler differentials in the commutative algebra framework we define for a divided powers algebra $A \in \mathrm{p}$-Com

(C) 2009 Australian Mathematical Publishing Association Inc. 1446-7887/2009 \$16.00 
the module of differentials $\Omega_{\mathrm{p}}^{A}$ which represents the Beck derivations. Moreover, we construct the abelianization functor

$$
\mathrm{Ab}: \mathrm{p}-\mathrm{Com} / A \rightarrow(\mathrm{p}-\mathrm{Com} / A)_{\mathrm{ab}}
$$

left adjoint to the forgetful functor

$$
F:(\mathrm{p}-\operatorname{Com} / A)_{\mathrm{ab}} \rightarrow \mathrm{p}-\mathrm{Com} / A \text {. }
$$

In Section 5, using the general scheme of the Quillen-Barr-Beck theory (see [1, 6]) for cohomology for universal algebras, we define cohomology groups $H^{*}(A, M)$ for a divided powers algebra $A \in \mathrm{p}$-Com and a Beck $A$-module $M$. Also, we define extensions and we give an interpretation of the first cohomology $H^{1}$ in terms of extensions. Precisely, we prove a classification theorem which give us a bijection between the first cohomology and the set of equivalence classes of extensions.

\section{Divided powers algebras}

The notion of commutative algebras with divided powers was introduced by Cartan in [2]. Also, we note that divided power algebras were studied by Roby in [7, 8]. Let us recall the definition of divided powers algebra.

Let $\mathcal{R}$ be a commutative ring. We denote by Com the category of commutative algebras over $\mathcal{R}$. Let $A$ be an augmented commutative algebra over $\mathcal{R}$ with unit 1 and with augmentation ideal $A_{+}$.

Definition 2.1. A commutative augmented algebra $A$ over $\mathcal{R}$ is called an algebra with divided powers if there are operations $\gamma_{i}: A_{+} \rightarrow A$, for $i \geq 0$, such that the following relations hold:

$$
\begin{gathered}
\gamma_{0}(x)=1, \quad \gamma_{1}(x)=x, \quad \gamma_{i}(x) \in A_{+}, \quad x \in A_{+}, \quad i \geq 1 \\
\gamma_{i}(x+y)=\sum_{k=0}^{i} \gamma_{k}(x) \gamma_{i-k}(y), \quad x, y \in A_{+}, \quad i \geq 0 \\
\gamma_{i}(a x)=a^{i} \gamma_{i}(x), \quad a \in A, \quad x \in A_{+}, \quad i \geq 0 \\
\gamma_{i}(x) \gamma_{j}(x)=\frac{(i+j) !}{i ! j !} \gamma_{i+j}(x), \quad x \in A_{+}, \quad i, j \geq 0 \\
\gamma_{i}\left(\gamma_{j}(x)\right)=\frac{(i j) !}{i !(j !)^{i}} \gamma_{i j}(x), \quad x \in A_{+}, \quad i \geq 0, \quad j \geq 1
\end{gathered}
$$

EXAMPLE 1. If the ring $\mathcal{R}$ is a field of characteristic zero, then any commutative graded algebra $A$ can be equipped with a unique system of divided powers given by $\gamma_{i}(x):=x^{i} / i$ ! for all $x \in A_{+}$and $i \geq 0$.

EXAMPLE 2. The homotopy of a simplicial commutative $\mathcal{R}$-algebra is equipped with the structure of divided powers algebra [2]. 
The next example is related with the notion of Zinbiel algebra introduced by Loday [4,5]. A Zinbiel algebra $(G, \prec)$ is an $\mathcal{R}$-module equipped with a binary operation $\prec$ such that

$$
(x \prec y) \prec z=x \prec(y \prec z)+x \prec(z \prec y)
$$

for all $x, y \in G$. Any Zinbiel algebra $G$ is endowed with the structure of a commutative algebra by defining a new operation $\star$ given by

$$
x \star y:=x \prec y+y \prec x
$$

for all $x, y \in G$. Therefore, we have a functor Zinb $\rightarrow$ Com from the category of Zinbiel algebras to the category of commutative algebras.

Example 3. Let $(G, \prec)$ be a unital Zinbiel algebra over $\mathcal{R}$. Then $\left(G, \star, \gamma_{i}\right)$ is a commutative algebra with divided powers where the $\gamma_{i}(x)$ are defined inductively by $\gamma_{1}(x)=x$ and $\gamma_{i}(x)=x \prec \gamma_{i-1}(x)$.

ExAmple 4. From Example 3, we see that the structure of divided powers arise naturally whenever there is a Zinbiel algebra structure. Let $M$ be a $\mathcal{R}$-module then the tensor module $T(M)$ equipped with the half-shuffle product is a Zinbiel algebra [5]. Therefore $T(M)$ can be endowed with the structure of a commutative algebra with divided powers.

2.1. Free divided powers algebra If $M$ is a free $\mathcal{R}$-module, then the free commutative algebra with divided powers on $M$ is the symmetric tensor algebra $F(M)=\bigoplus_{n}\left(M^{\otimes n}\right)^{S_{n}}$. If $t_{n} \in\left(M^{\otimes n}\right)^{S_{n}}$, then the system of divided powers is given by

$$
\gamma_{k}\left(t_{n}\right):=\sum_{\sigma \in K_{n, k}} \sigma\left(t_{n}^{\otimes k}\right),
$$

where

$$
K_{n, k}:=\left\{\sigma \in S_{n, k} \mid \sigma(n)<\sigma(2 n)<\cdots<\sigma(k n)\right\}
$$

and $S_{n, k}$ denotes the set of $(\underbrace{n, n, \ldots, n}_{k})$-shuffles (for details see $[8,2,3]$ ).

2.2. Divided powers algebras in prime characteristic Now, we suppose that the ground ring is the field $k=\mathbb{F}_{p}$. We denote by $\mathrm{p}$-Com the category of commutative $\mathbb{F}_{p^{-}}$ algebras with divided powers. In prime characteristic, by a theorem of Cartan in [2], we have that the system of divided powers of a divided power algebra $A \in \mathrm{p}$-Com is induced by the operation $\gamma_{p}$. Moreover, let $A$ be an augmented $\mathbb{F}_{p}$-algebra such that $x^{p}=0$ for all $x \in A_{+}$, together with a map $\pi: A_{+} \rightarrow A_{+}$which verifies the relations of the $p$ th divided power. Then by Theorem 1 of Soublin [9], $A$ is equipped with a unique system of divided powers with $\gamma_{p}=\pi$.

Considering these facts in prime characteristic, it follows from the relations of Definition 2.1 that the category p-Com is equivalent to a category whose objects are 
commutative augmented algebras $(A, \pi)$ together with a map $\pi: A_{+} \rightarrow A_{+}$which verifies the following relations:

$$
\begin{gathered}
x^{p}=0, \\
\pi(x+y)=\pi(x)+\pi(y)+\sum_{k=1}^{p-1} \frac{(-1)^{k}}{k} x^{k} y^{(p-k)}, \\
\pi(x y)=0, \\
\pi(\lambda x)=\lambda^{p} \pi(x),
\end{gathered}
$$

where $x, y \in A_{+}$and $\lambda \in k$.

Moreover, we note that in $\mathrm{p}$-Com the morphisms $f$ are homomorphisms of commutative rings such that $f \pi=\pi f$.

\section{Beck modules and derivations}

In order to define cohomology in p-Com we follow the general scheme of cohomology theories for universal algebras due to Quillen [6] and Barr and Beck [1]. In particular, we have to determine for a divided powers algebra $A$ the category of abelian group objects over $A$, or in other words the category of Beck $A$-modules.

Let $(A, \pi) \in \mathrm{p}$-Com be a divided powers algebra. We denote by $\mathrm{p}-\mathrm{Com} / A$ the slice category over $A$ and by (p-Com/ $A)_{\text {ab }}$ the category of abelian group objects in p-Com $/ A$. Let $(M, \pi)$ be an $A$-module together with a $p$-semilinear map $\pi: M \rightarrow M$ such that $\pi(\mathrm{am})=0$ for all $a \in A_{+}$and $m \in M$. We consider $A \oplus M$ the semidirect product commutative $A$-algebra with multiplication given by

$$
\left(a_{1}, m_{1}\right)\left(a_{2}, m_{2}\right)=\left(a_{1} a_{2}, a_{1} m_{2}+a_{2} m_{1}\right) .
$$

We define a map $\pi: A \oplus M \rightarrow A \oplus M$ given by

$$
\pi((a, m))=\left(\pi(a), \pi(m)-a^{p-1} m\right) .
$$

The commutative algebra $A \oplus M$ together with the above map

$$
\pi: A \oplus M \rightarrow A \oplus M
$$

are denoted by $A \oplus_{p} M$.

LEMMA 3.1. Let $(A, \pi)$ be a divided powers algebra and let $(M, \pi)$ be an A-module together with a p-semilinear map $\pi: M \rightarrow M$ such that $\pi(\mathrm{am})=0$ for all $a \in A_{+}$ and $m \in M$. Then $A \oplus_{p} M$ is a divided powers algebra. 
PRoOF. In $\operatorname{char}(k)=p$ we have $(a, m)^{p}=\left(a^{p}, p a^{p-1} m\right)=(0,0)$, and therefore relation (6) is true. For relation (7)

$$
\begin{aligned}
\pi & ((a, m))+\pi((b, n))+\sum_{k=1}^{p-1} \frac{(-1)^{k}}{k}(a, m)^{k}(b, n)^{p-k} \\
= & \left(\pi(a), \pi(m)-a^{p-1} m\right)+\left(\pi(b), \pi(n)-b^{p-1} n\right) \\
& +\sum_{k=1}^{p-1} \frac{(-1)^{k}}{k}(a, m)^{k}(b, n)^{p-k}
\end{aligned}
$$

where $a, b \in A_{+}$and $m, n \in M$. In addition,

$$
\begin{aligned}
\sum_{k=1}^{p-1} & \frac{(-1)^{k}}{k}(a, m)^{k}(b, n)^{p-k} \\
= & \sum_{k=1}^{p-1} \frac{(-1)^{k}}{k}\left(a^{k}, k a^{k-1} m\right)\left(b^{p-k},(p-k) b^{p-k-1} n\right) \\
= & \sum_{k=1}^{p-1} \frac{(-1)^{k}}{k}\left(a^{k} b^{p-k},\left(a^{k}(p-k) b^{p-k-1}\right) n+\left(b^{p-k} k a^{k-1}\right) m\right) \\
= & \left(\sum_{k=1}^{p-1} \frac{(-1)^{k}}{k} a^{k} b^{p-k},\left(\sum_{k=1}^{p-2}(-1)^{k+1} a^{k} b^{p-k-1}\right) n-a^{p-1} n\right. \\
& \left.+\left(\sum_{k=2}^{p-1}(-1)^{k} a^{k-1} b^{p-k}\right) m-b^{p-1} m\right) \\
= & \left(\sum_{k=1}^{p-1} \frac{(-1)^{k}}{k} a^{k} b^{p-k},\left(\sum_{k=1}^{p-2}(-1)^{k+1} a^{k} b^{p-k-1}\right)(m+n)-a^{p-1} n-b^{p-1} m\right) .
\end{aligned}
$$

Therefore, we obtain

$$
\begin{aligned}
& \pi((a, m))+\pi((b, n))+\sum_{k=1}^{p-1} \frac{(-1)^{k}}{k}(a, m)^{k}(b, n)^{p-k} \\
& =\left(\pi(a)+\pi(b)+\sum_{k=1}^{p-1} \frac{(-1)^{k}}{k} a^{k} b^{p-k}, \pi(m)+\pi(n)\right. \\
& \left.\quad+\left(\sum_{k=0}^{p-1}(-1)^{k+1} a^{k} b^{p-k-1}\right)(m+n)\right) .
\end{aligned}
$$


Since in characteristic $p$ the identity $\left(\begin{array}{c}p-1 \\ k\end{array}\right) \equiv(-1)^{k} \bmod p$ holds if $0 \leq k<p$, it follows that

$$
\begin{aligned}
& \pi((a, m))+\pi((b, n))+\sum_{k=1}^{p-1} \frac{(-1)^{k}}{k}(a, m)^{k}(b, n)^{p-k} \\
& \quad=\left(\pi(a+b), \pi(m+n)-(a+b)^{p-1}(m+n)\right) \\
& \quad=\pi((a+b, m+n)) .
\end{aligned}
$$

For relation (8),

$$
\begin{aligned}
\pi((a, m)(b, n)) & =\pi((a b, a n+b m)) \\
& =\left(\pi(a b), \pi(a n+b m)+(a b)^{p-1}(a n+b m)\right) \\
& =\left(\pi(a b), \pi(a n)+\pi(b m)+\left(b^{p-1} a^{p}\right) n+\left(a^{p-1} b^{p}\right) m\right) \\
& =(0,0) .
\end{aligned}
$$

Finally, relation (9) is obviously true.

Let $(A, \pi)$ be a divided powers algebra and let $(M, \pi)$ be an $A$-module together with a $p$-semilinear map $\pi: M \rightarrow M$ such that $\pi(\mathrm{am})=0$ for all $a \in A_{+}$and $m \in M$. Then if $\operatorname{Der}(A, M)$ denotes the group of derivations of the commutative algebra $A$ with values in the $A$-module $M$, we denote by $\operatorname{Der}(A,(M, \pi))$ the following abelian group:

$\operatorname{Der}(A,(M, \pi))=\left\{D \in \operatorname{Der}(A, M) \mid D(\pi(a))=\pi(D(a))-a^{p-1} D(a), a \in A_{+}\right\}$.

We note that $\operatorname{Der}(A,(M, \pi))$ has the structure of a group since $\pi: M \rightarrow M$ is a $p$-semilinear map on $M$. We consider $A \oplus_{p} M$ as an object in p-Com/ $A$ via the projection $A \oplus_{p} M \rightarrow A$.

TheOREM 3.2. Let $(A, \pi)$ be a divided powers algebra and let $(M, \pi)$ be an $A$-module together with a $p$-semilinear map $\pi: M \rightarrow M$ such that $\pi($ am $)=0$ for all $a \in A_{+}$and $m \in M$. Then for all $B \in \mathrm{p}-\mathrm{Com} / A$, we have the following isomorphism of groups:

$$
\operatorname{Hom}_{\mathrm{p}-\mathrm{Com} / A}\left(B, A \oplus_{p} M\right) \simeq \operatorname{Der}(B,(M, \pi))
$$

where we view $M$ as a $B$-module via the morphism $f: B \rightarrow A$.

Proof. Let $g: B \rightarrow A \oplus_{p} M$ be an homomorphism over $A$. Then the first coordinate of $g$ must be $f$. If we denote by $d_{g}$ the second coordinate of $g$, then $d_{g}$ is linear because $g$ is. Since $g$ is a commutative homomorphism, it follows that

$$
d_{g}\left(b_{1} b_{2}\right)=f\left(b_{1}\right) d_{g}\left(b_{2}\right)+f\left(b_{2}\right) d_{g}\left(b_{1}\right)
$$

where $b_{1}, b_{2} \in B$. The fact that $g$ is a divided powers homomorphism implies that

$$
d_{g}(\pi(b))=\pi\left(d_{g}(b)\right)-f(b)^{p-1} d_{g}(b),
$$

where $b \in B_{+}$. 
Conversely, let $d \in \operatorname{Der}(B,(M, p))$ and let $g_{d}: B \rightarrow A \oplus_{p} M$ be the map given by $g_{d}:=(f, d)$. We have

$$
\begin{aligned}
g_{d}\left(b_{1} b_{2}\right) & =\left(f\left(b_{1} b_{2}\right), d\left(b_{1} b_{2}\right)\right) \\
& =\left(f\left(b_{1}\right) f\left(b_{2}\right), f\left(b_{1}\right) d\left(b_{2}\right)+f\left(b_{2}\right) d\left(b_{1}\right)\right) \\
& =\left(f\left(b_{1}\right), d\left(b_{1}\right)\right)\left(f\left(b_{2}\right), d\left(b_{2}\right)\right) \\
& =g_{d}\left(b_{1}\right) g_{d}\left(b_{2}\right),
\end{aligned}
$$

and therefore $g_{d}$ is a commutative homomorphism over $A$. Moreover,

$$
\begin{aligned}
g_{d}(\pi(b)) & =(f(\pi(b), d(\pi(b))) \\
& =\left(\pi(f(b)), \pi(d(b))-f(b)^{p-1} d(b)\right) \\
& =\pi\left(g_{d}(b)\right)
\end{aligned}
$$

for all $b \in B_{+}$. Thus, $g_{d}$ is a divided powers homomorphism. Finally, we easily see that $d_{\left(g_{d}\right)}=d$ and $g_{\left(d_{g}\right)}=g$. The maps $\phi: g \rightarrow d_{g}$ and $\psi: d \rightarrow g_{d}$ are isomorphisms of groups such that $\phi \psi=\psi \phi=$ Id.

REMARK 1. Theorem 3.2 above implies that $A \oplus_{p} M \rightarrow A$ is an abelian group object in $\mathrm{p}-\mathrm{Com} / A$.

Moreover, we have the following theorem.

THEOREM 3.3. Let $A \in \mathrm{p}$-Com be a commutative divided powers algebra and let $B \in(\mathrm{p}-\mathrm{Com} / A)_{\mathrm{ab}}$ be an abelian group object. Then there exists an isomorphism of divided powers algebras

$$
B \simeq A \oplus_{p} M
$$

for a specific pair $(M, \pi)$, where $M$ is an A-module and $\pi: M \rightarrow M$ a p-semilinear map such that $\pi(a m)=0, a \in A_{+}$.

Proof. Let $f: B \rightarrow A$ be the map over $A$ with kernel $M:=\operatorname{ker} f$. From the group structure there exists a divided power morphism $z: A \rightarrow B$ which splits $f$. Therefore, as $k$-modules there is an isomorphism $\omega: A \oplus M \rightarrow B$ given by

$$
(a, m):=z(a)+m a \in A, \quad m \in M,
$$

where + denotes addition in $B$. The action $a m:=z(a) m$ endows $M$ with the structure of $A$-module such that $\pi(a m)=0$ for all $a \in A_{+}$and $m \in M$. Since $B$ is an abelian group object in Com/A we obtain that actually $\omega$ is a commutative algebra isomorphism where the multiplication in $A \oplus M$ is given by

$$
\left(a_{1}, m_{1}\right)\left(a_{2}, m_{2}\right)=\left(a_{1} a_{2}, a_{1} m_{2}+a_{2} m_{1}\right) .
$$


In addition,

$$
\begin{aligned}
\pi(\omega(a, m)) & =\pi(z(a)+m) \\
& =\pi(z(a))+\pi(m)+\sum_{k=1}^{p-1} \frac{(-1)^{k}}{k}(z(a))^{k} m^{p-k} \\
& =z(\pi(a))+\pi(m)-z(a)^{p-1} m \\
& =\omega\left(\pi(a), \pi(m)-a^{p-1} m\right) \\
& =\omega(\pi(a, m)) .
\end{aligned}
$$

It follows that the corresponding divided power algebra $A \oplus_{p} M$ to the pair $(M, \pi)$ is isomorphic to $B$.

We denote by $k[P]$ the noncommutative polynomial ring defined by

$$
k[P]:=\left\{\sum_{i=0}^{n} \lambda_{i} P^{i} \mid P \lambda_{i}=\lambda_{i}^{p} P, i, n \in \mathbb{N}, \lambda_{i} \in k\right\} .
$$

Let $A$ be a divided power algebra. We define $V(A)$ to be the ring whose underlying $k$-module is $V(A):=A \otimes_{k} k[P]$ and whose multiplication is given by

$$
\begin{gathered}
\left(a_{1} \otimes 1\right)\left(a_{2} \otimes 1\right)=\left(a_{1} a_{2} \otimes 1\right), \quad a_{1}, a_{2} \in A, \\
\left(1 \otimes q_{1}\right)\left(1 \otimes q_{2}\right)=\left(1 \otimes q_{1} q_{2}\right), \quad q_{1}, q_{2} \in k[P], \\
(a \otimes 1)(1 \otimes P)=(a \otimes P), \quad a \in A, \\
(1 \otimes P)(a \otimes 1)=0, \quad a \in A_{+}, \\
(1 \otimes P)(\lambda \otimes 1)=\left(\lambda^{p} \otimes P\right), \quad \lambda \in k .
\end{gathered}
$$

THEOREM 3.4. Let $A$ be a divided powers algebra. Then the category $(\mathrm{p}-\mathrm{Com} / A)_{\mathrm{ab}}$ is equivalent to the category of left $V(A)$-modules.

PROOF. Any abelian group object is determined by a pair $(M, \pi)$ where $M$ is an $A$-module and $\pi: M \rightarrow M$ a $p$-semilinear map such that $\pi(\mathrm{am})=0$ for all $a \in A_{+}$and $m \in M$. We endow $M$ with the structure of a $V(A)$-module as follows: $(1 \otimes P) m:=\pi(m)$ and $(a \otimes 1) m:=a m$ for all $a \in A$. With this $V(A)$-module structure, $M$ will be denoted by $\mathcal{M}$. Therefore, we associated to each pair $(M, \pi)$ a $V(A)$-module $\mathcal{M}$.

Conversely, to each $V(A)$-module $\mathcal{M}$ we associate a pair $(M, \pi)$ with $\pi: M \rightarrow M$ such that $\pi(\mathrm{am})=0$ for all $a \in A_{+}$and $m \in M$. We consider $\mathcal{M}$ as $A$-module $M$ with action given by $a m:=(a \otimes 1)$ and we define $\pi: M \rightarrow M$ by $\pi(m):=(1 \otimes P) m$ for all $a \in A$ and $m \in M$.

Notation 3.5. Let $A$ be a divided powers algebra and let $\mathcal{M}$ be a $V(A)$-module. From the equivalence of Theorem 3.4 we have that $\mathcal{M}$ is associated to a pair $(M, \pi)$ where $M$ is an $A$-module and $\pi: M \rightarrow M$ is a $p$-semilinear map such that $\pi(\mathrm{am})=0$ for all $a \in A_{+}$and $m \in M$. We adopt the following notation:

$$
\operatorname{Der}_{p}(A, \mathcal{M}):=\operatorname{Der}(A,(M, \pi)) .
$$


REMARK 2. Let $A$ be a divided powers algebra. An abelian group object $\mathcal{M} \in$ $(\mathrm{p}-\mathrm{Com} / A)_{\mathrm{ab}}$ is called a Beck $A$-module, in the light of the work of Beck [1]. By Theorems 3.2, 3.3, 3.4 the group $\operatorname{Der}_{p}(A, \mathcal{M})$ is actually the group of derivations in Beck's sense of $A$ with values in the Beck $A$-module $\mathcal{M}$.

\section{The abelianization functor}

4.1. The differentials Let $(A, \pi)$ be a divided powers algebra and let $\mathcal{M}$ be a $V(A)$ module. We define $P m:=(1 \otimes P) m$ and $a m:=(a \otimes 1) m$ where $a \in A$ and $m \in \mathcal{M}$. We define $\Omega_{\mathrm{p}}^{A}$ to be the $V(A)$-module with the following presentation: the generators are the symbols $d a$ for $a \in A$ and the relations are

$$
\begin{gathered}
d(\lambda a+\mu b)=\lambda d a+\mu d b \\
d(a b)=a d b+b d a \\
d(\pi(c))=P d(c)-c^{p-1} d c
\end{gathered}
$$

where $a, b \in A, c \in A_{+}$.

THEOREM 4.1. Let $A$ be a divided powers algebra and let $\mathcal{M}$ be a $V(A)$-module. Then we have the following:

$$
\operatorname{Der}_{p}(A, \mathcal{M}) \simeq \operatorname{Hom}_{V(A)}\left(\Omega_{\mathrm{p}}^{A}, \mathcal{M}\right) .
$$

ProOf. Let $f: \Omega_{\mathrm{p}}^{A} \rightarrow \mathcal{M}$ be a $V(A)$ homomorphism. Then we associate to $f$ the map $D_{f}: A \rightarrow \mathcal{M}$ given by $D_{f}(a):=f(d a)$. Since $f$ is a $V(A)$-homomorphism from the relations (10), (11), (12) above it follows that $D_{f} \in \operatorname{Der}_{p}(A, \mathcal{M})$ and $D_{f}$ is a derivation in Beck's sense.

Conversely, let $D \in \operatorname{Der}_{p}(A, \mathcal{M})$ be a Beck derivation and let $\mathcal{F}$ be the free $V(A)$-module generated by the symbols $d a$ for all $a \in A$. We consider the $V(A)$ homomorphism $f_{D}^{\prime}: \mathcal{F} \rightarrow \mathcal{M}$ given by $f_{D}^{\prime}(d a):=D(a)$. Then

$$
\begin{aligned}
f_{D}^{\prime}(d(a b)) & =D(a b) \\
& =a D(b)+b D(a) \\
& =a f_{D}^{\prime}(d b)+b f_{D}^{\prime}(d a) \\
& =f_{D}^{\prime}(a d b+b d a)
\end{aligned}
$$

and

$$
\begin{aligned}
f_{D}^{\prime}(d \pi(a)) & =D(\pi(a)) \\
& =P D(a)-a^{p-1} D(a) \\
& =P f_{D}^{\prime}(d a)-a^{p-1} f_{D}^{\prime}(d a) \\
& =f_{D}^{\prime}\left(P d a-a^{p-1} d a\right) .
\end{aligned}
$$

Therefore, $f_{D}^{\prime}$ induce an homomorphism $f_{D}: \Omega_{\mathrm{p}}^{A} \rightarrow \mathcal{M}$. The maps $f \longmapsto f_{D}$ and $D \longmapsto D_{f}$ are inverse to each other and the theorem follows. 
4.2. The abelianization functor Let $A$ be divided powers algebra, let $B \in \mathrm{p}$-Com $/ A$ and let $\mathcal{M}$ be a $V(A)$-module with associated pair $(M, \pi)$ (see Theorem 3.4). Then we have the following isomorphisms:

$$
\begin{aligned}
\operatorname{Hom}_{(\mathrm{p}-\mathrm{Com} / A)}\left(B, A \oplus_{p} M\right) & \simeq \operatorname{Der}_{p}(B, \mathcal{M}) \\
& \simeq \operatorname{Hom}_{V(B)}\left(\Omega_{\mathrm{p}}^{B}, \mathcal{M}\right) \\
& \simeq \operatorname{Hom}_{V(A)}\left(V(A) \otimes_{V(B)} \Omega_{\mathrm{p}}^{B}, \mathcal{M}\right) .
\end{aligned}
$$

Therefore, the abelianization functor

$$
\mathrm{Ab}: \mathrm{p}-\mathrm{Com} / A \rightarrow(\mathrm{p}-\mathrm{Com} / A)_{\mathrm{ab}}
$$

left adjoint to the forgetful functor

$$
F:(\mathrm{p}-\mathrm{Com} / A)_{\mathrm{ab}} \rightarrow \mathrm{p}-\mathrm{Com} / A
$$

is given by

$$
\mathrm{Ab}: B \mapsto V(A) \otimes_{V(B)} \Omega_{\mathrm{p}}^{B}
$$

\section{5. (Co-)homology for divided powers algebras in prime characteristic}

Let $A$ be a divided powers algebra and let $\mathcal{M}$ be a $V(A)$-module. We choose a simplicial cofibrant resolution $Q$ of $A$ (see Remark 3 below). Following Quillen's notation we denote by $\operatorname{LAb}(A)$ the simplicial object of $(\mathrm{p}-\mathrm{Com} / A)_{\mathrm{ab}}$ given by $\mathrm{LAb}(A):=\mathrm{Ab}(Q)$. According to Quillen's definition of cohomology of universal algebras (see [6]) the cohomology groups of $A$ with values in $\mathcal{M}$ are given by the formula

$$
\mathrm{H}^{n}(A, \mathcal{M}):=\mathrm{H}^{n}\left(\operatorname{Hom}_{(\mathrm{p}-\mathrm{Com} / A)_{\mathrm{ab}}}(\mathbf{L A b}(A), \mathcal{M})\right)
$$

where on the right-hand side of the formula, $\mathrm{H}^{n}$ denotes the cohomology of a cosimplicial abelian group. Moreover, the $n$th homology object of $A$ is given by

$$
\mathrm{H}_{n}(A):=\mathrm{H}_{n}(\mathbf{L A b}(A)) .
$$

Therefore, considering the isomorphism of Theorem 3.2, we make the following definition.

Definition 5.1. Let $A$ be a divided powers algebra and let $\mathcal{M}$ be a $V(A)$-module. Then the cohomology of $A$ with values in $\mathcal{M}$ is given by

$$
\mathrm{H}^{n}(A, \mathcal{M}):=\mathrm{H}^{n}\left(\operatorname{Der}_{p}(Q, \mathcal{M})\right),
$$

where in the right-hand side of the formula we denote the cohomology of the cosimplicial object $\operatorname{Der}_{p}(Q, \mathcal{M})$. In addition, the homology of $A$ is given by

$$
\mathrm{H}_{n}(A):=\mathrm{H}_{n}\left(V(A) \otimes_{V(Q)} \Omega_{\mathrm{p}}^{Q}\right),
$$

where, in the right-hand side of the formula, $\mathrm{H}_{n}$ denotes the homology of a simplicial object. 
REMARK 3. Since we have free objects we can obtain a simplicial cofibrant resolution $Q$ from the cotriple induced by the adjunction of the free functor and the forgetful functor $U$ from the category of divided powers algebras to the category of $\mathcal{R}$-modules.

REMARK 4. It follows that $\mathrm{H}^{0}(A, \mathcal{M})=\operatorname{Der}_{p}(A, \mathcal{M})$.

5.1. Extensions In this section we give an interpretation of the first cohomology group in terms of extensions. Next we define extensions in p-Com. Here, we note that in the interpretations of cohomology in low dimensions $\left(\mathrm{H}^{0}, \mathrm{H}^{1}\right)$ there is a shift in the dimension in the Quillen-Barr-Beck definition compared with the usual notation.

Definition 5.2. Let $A$ be a divided powers algebra and let $\mathcal{M}$ be a $V(A)$-module. Then an extension of $A$ by $\mathcal{M}$ is a short exact sequence of divided powers algebras:

$$
(\mathcal{E}): \quad 0 \rightarrow \mathcal{M} \rightarrow E \rightarrow A \rightarrow 0,
$$

where $\mathcal{M}^{2}=0$ and such that the given $V(A)$-module structure in $\mathcal{M}$ agrees with that induced by the extension.

Two such extensions $\mathcal{E}_{1}$ and $\mathcal{E}_{2}$ of $A$ by $\mathcal{M}$ are called equivalent if there is an isomorphism $f: \mathcal{E}_{1} \rightarrow \mathcal{E}_{2}$ such that the following diagram is commutative.

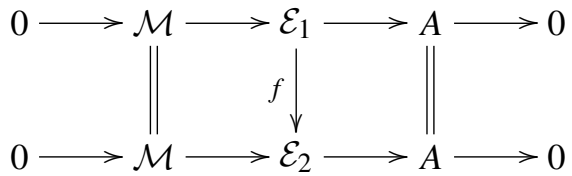

We denote by $E(A, \mathcal{M})$ the set of equivalence classes of extensions of $A$ by $\mathcal{M}$.

THEOREM 5.3. Let $A$ be a divided powers algebra and let $\mathcal{M}$ be a $V(A)$-module. Then there is a one-to-one correspondence between the set $E(A, \mathcal{M})$ of equivalences classes of extensions of $A$ by $\mathcal{M}$ and the first cohomology group $H^{1}(A, \mathcal{M})$.

PROOF. We recall that by Theorem 3.4, the $V(A)$-module $\mathcal{M}$ is determined by a pair $(M, \pi)$ where $M$ is an $A$-module and $\pi: M \rightarrow M$ is a $p$-semilinear map such that $\pi(a m)=0$ for all $a \in A_{+}, m \in M$. From [6, Proposition 2.4], the first cohomology group $H^{1}(A, \mathcal{M})$ is the isomorphism classes of objects $E \rightarrow A$ which are torsors for $A \oplus \oplus_{p} M \rightarrow A$.

Let $E \stackrel{f}{\rightarrow} A$ be a torsor object for $A \oplus_{p} M \rightarrow A$. Then $E \rightarrow A$ is an epimorphism and there is an action

$$
\alpha:\left(A \oplus_{p} M\right) \times_{A} E \rightarrow E
$$

such that the map

$$
(\alpha, p):\left(A \oplus_{p} M\right) \times_{A} E \rightarrow E \times_{A} E
$$

is an isomorphism, where $p$ denotes the projection. At this point, we consider the kernel $K:=\operatorname{ker}(E \rightarrow A)$ and the injection

$$
i: K \rightarrow E \times{ }_{A} E
$$


given by $i(k):=(k, 0)$. In addition, we have an injection

$$
j: M \rightarrow\left(A \oplus_{p} M\right) \times_{A} E
$$

given by $j(m):=((0, m), 0)$. Then from the restriction of $(\alpha, p)$ to $M$ we obtain a divided powers isomorphism $(\alpha, p) j: M \rightarrow K$. In addition, let $a \in A$ and $m \in M$. Then we have

$$
((\alpha, p) j)(a m)=(\alpha((0, a m), 0), 0) .
$$

Take $e_{a} \in E$ such that $f\left(e_{a}\right)=a$. Since $\alpha$ is a commutative homomorphism we have

$$
\begin{aligned}
\alpha((0, a m), 0) & =\alpha\left(\left((a, 0), e_{a}\right)((0, m), 0)\right) \\
& \left.=\alpha\left((a, 0), e_{a}\right) \alpha((0, m), 0)\right) .
\end{aligned}
$$

Moreover, from the fact that $(\alpha, p)$ is an isomorphism

$$
f\left(\alpha\left((a, 0), e_{a}\right)\right)=a .
$$

Therefore, we obtain

$$
((\alpha, p) j)(a m)=(a \alpha((0, m), 0)), 0)
$$

and $M$ and $K$ are isomorphic as $A$-modules. From the above, it follows that $M$ and $K$ are isomorphic as $V(A)$-modules as well. Conversely, let $\mathcal{E} \in E(A, \mathcal{M})$ be an extension of $A$ by $\mathcal{M}$

$$
\mathcal{E}: 0 \rightarrow \mathcal{M} \rightarrow E \stackrel{f}{\rightarrow} A \rightarrow 0
$$

Then $f: E \rightarrow A$ is a surjection. We consider the map

$$
\alpha:\left(A \oplus_{p} M\right) \times_{A} E \rightarrow E
$$

given by

$$
\alpha:((f(e), m), e) \mapsto e+m .
$$

It is easy to see that the map is well defined. Since $f$ is a divided powers homomorphism and the induced action of the extension agrees with the given one, $\alpha$ is a divided homomorphism. Therefore, $\alpha$ defines an action. In addition, the map

$$
(\alpha, p):\left(A \oplus_{p} M\right) \times_{A} E \rightarrow E \times_{p} E
$$

where $p$ denotes the projection, is well defined and is actually a divided powers isomorphism. Therefore, $E \rightarrow A$ is a torsor for $A \oplus_{p} M \rightarrow A$. 


\section{References}

[1] M. Barr and J. Beck, 'Homology and standard constructions', in: Seminar on Triples and Categorical Homology Theory, Lecture Notes in Mathematics, 80 (Springer, Berlin, 1969), pp. 245-335.

[2] H. Cartan, 'Algèbres de Eilenberg-MacLane et homotopie', in: Seminaire Henri Cartan, 7ème année 1954-1955, 2ème édn (École Normale Supérieure, Paris, 1956).

[3] B. Fresse, 'On the homotopy of simplicial algebras over an operad', Trans. Amer. Math. Soc. 352(9) (2000), 4113-4141.

[4] J.-L. Loday, 'Cup-product for Leibniz cohomology and dual Leibniz algebras', Math. Scand. 77 (1995), 189-196.

[5] — 'Dialgebras', in: Dialgebras and Related Operads, Lectures Notes in Math., 1763 (Springer, Berlin, 2001), pp. 7-66.

[6] D. Quillen, 'On the (co-)homology of commutative rings', in: Applications of Categorical Algebra, New York, 1968, Proc. Sympos. Pure Math., Vol. XVII (American Mathematical Society, Providence, RI, 1970), pp. 65-87.

[7] N. Roby, 'Les algèbres à puissances divisées', Bull. Soc. Math. France 89 (1965), 75-91.

[8] _ _Construction de certaines algèbres à puissances divisées', Bull. Soc. Math. France 96 (1968), 97-113.

[9] J.-P. Soublin, 'Puissances divisées en caractéristique non nulle', J. Algebra 110 (1987), 523-529.

IOANNIS DOKAS, Department of Mathematics and Statistics, University of Cyprus, PO Box 20537, CY-1678, Nicosia, Cyprus

e-mail: dokas@ucy.ac.cy 